Published in final edited form as:

Autoimmun Rev. 2010 February ; 9(4): . doi:10.1016/j.autrev.2009.07.004.

\title{
Malignancies in systemic lupus erythematosus
}

\author{
Mruganka Kale ${ }^{1}$, Rosalind Ramsey-Goldman ${ }^{2}$, Caroline Gordon ${ }^{3}$, Ann E Clarke ${ }^{1}$, and Sasha \\ Bernatsky ${ }^{*}, 1$ \\ ${ }^{1}$ McGill University Health Centre, 687 Pine Avenue West, Montreal, Quebec, H3A 1A1, Canada \\ ${ }^{2}$ Division of Rheumatology, Feinberg School of Medicine, Northwestern University, Chicago, IL, \\ USA
}

${ }^{3}$ Department of Rheumatology, University of Birmingham, Edgbaston, Birmingham, UK

\begin{abstract}
The purpose of this review is to underline important advancements in the understanding of cancer risks in systemic lupus erythematosus (SLE). In SLE, there is an increased risk of specific kinds of malignancy. For example, the risk of non-Hodgkin's lymphoma is increased several-fold in SLE versus the general population. In addition, heightened risks for lung cancer, thyroid cancer and cervical dysplasia in SLE have been found. Some have postulated that immunosuppressive drugs play a role, as well as other important mediators, such as lupus disease activity itself. One new frontier being explored is the significant finding of a decreased risk of certain nonhematologic cancers (e.g., breast, ovarian, endometrial and prostate) in SLE. The reasons for this are currently under study.
\end{abstract}

\section{Keywords}

cancer; immunosuppressive drug; malignancy; risk; systemic lupus erythematosus

In systemic lupus erythematosus (SLE), there appears to be specific differences in cancer susceptibility, compared with the general population. To date, data indicate an overall increase in cancer in SLE (10-15\%) [1], with definite increases in hematologic cancers, particularly non-Hodgkin's lymphoma (NHL) as well as lung, thyroid and vulvar cancer [14]. Cervical dysplasia (a precancerous lesion) is another condition of potential concern.

It has been postulated that immunosuppressive drugs may play a role in increased cancer risk in SLE, but there may also be other important factors, including SLE activity itself. According to recent findings, the risk for certain nonhematologic cancers, such as breast, endometrial and ovarian cancers in women [5], and prostate cancers in men, may be decreased in individuals with SLE [6,7]. Exploring the reasons for this is itself a whole new avenue of potential research, as will be discussed.

\footnotetext{
(C) 2013 Future Medicine Ltd

*Author for correspondence: Tel.: +1 5149341934 ext. 44710, sasha.bernatsky@ mail.mcgill.ca.

For reprint orders, please contact: reprints@futuremedicine.com
}

Financial \& competing interests disclosure

The authors have no relevant affiliations or financial involvement with any organization or entity with a financial interest in or financial conflict with the subject matter or materials discussed in the manuscript. This includes employment, consultancies, honoraria, stock ownership or options, expert testimony, grants or patents received or pending, or royalties.

No writing assistance was utilized in the production of this manuscript. 
This review will touch briefly on these topics, in order to summarize for readers, the emerging evidence regarding cancer risk and risk factors, in SLE.

\section{Hematologic cancers}

The heightened risk of lymphoma in SLE has been widely reported in many studies [1-4], with estimates fairly consistently suggesting a two- to three-fold increased incidence of NHL, compared with age- and sex-matched general population comparators. It should be noted that patients with SLE have a higher mortality rate owing to NHL than is seen in the general population, with a standardized mortality ratio (SMR) of 2.8 (95\% CI: 1.2-5.6) [8], compared with an age- and sex-matched general population. Individuals with SLE have also been found to suffer from higher risks of other types of hematological malignancies, such as Hodgkin's lymphoma [9] and leukemia (standardized incidence ratio [SIR]: 1.69; 95\% CI: 1.00-2.67) [2,3]. To date, estimates for the relative risk of multiple myeloma in SLE have not been conclusive; although one study found that monoclonal gammopathy (a precursor to multiple myeloma) was more frequent in SLE patients than the general population [10].

One mechanism for lymphoma development may involve the translocation of an oncogene beside a gene important for cell proliferation [11], which can favor the progression of a lymphoma. This may be important in individuals with SLE, who can have uncontrolled Bcell proliferation and/or defected clearance of apoptotic cells. However, at this time, that hypothesis remains unproven. The etiology of the increased risk for hematologic cancers in SLE, thus, remains under study.

It is well known that the immune system can both promote and suppress cancer. Chronic inflammation may certainly play a role in carcinogenesis in humans. By contrast, anti-tumor immunity remains an important way that humans protect against cancer development, although for many cancers, the exact mechanisms remain to be elucidated. Interestingly, a recent study in mice suggested that eradication of precursor myeloma and B-cell lymphoma cells occur via interactions between tumor-specific Th1 cells and tumor-infiltrating, antigenpresenting macrophages [12].

A recent study in Italy indicated that SLE disease activity and the course of lymphoma might not be directly related. A select group of patients with SLE who developed lymphoma were successfully treated with high-dose chemotherapy for lymphoma yet their SLE persisted or relapsed [13]. Recent and ongoing studies will hopefully determine the extent to which immunosuppressive exposures play a role, specifically in relation to other factors, including disease activity itself. The topic of medication exposures and cancer risk will be further described later in this review.

\section{Lung cancer}

Many cohort studies have shown that individuals with SLE have a heightened risk of lung cancer [4,5]. In our multicenter cohort study, the SIR for lung cancer was 1.24 (95\% CI: $1.00-1.53$ ) [5]. A Swedish study examining patients who had been hospitalized for any of 33 autoimmune disorders $(\mathrm{n}=402,062)$, of which 7624 were SLE patients, found an SIR of 2.47 (95\% CI: 1.97-3.05) and SMR of 2.69 (95\% CI: 0.98-1.54) for lung cancer in patients with SLE, affecting all lung cancer histologies, with the highest risk for small-cell carcinoma [14]. Case-cohort analyses performed by our group have confirmed that smoking is an important predictor of lung cancer in SLE. One study showed that the majority of SLE patients who developed lung cancers were smokers; however, only a small number had previously been exposed to immunosuppressive drugs [15]. This study also demonstrated a potential increase in the incidence of rare cancer (including bronchoalveolar and carcinoid) 
with comparable histological distribution of cancers in the SLE group and general population.

One small European study evaluated patients with systemic autoimmune rheumatic disease, including six SLE patients who developed lung cancer and were diagnosed at an advanced stage and had poor survival [16]. Although the authors encourage more efforts towards early detection of these cancers, it is not clear that a screening program should be put in place (since the benefits are not actually proven), although attention to risk factors (primarily, smoking) should certainly be prioritized.

\section{Thyroid cancers}

Although the etiology is yet to be confirmed, an increased risk of thyroid cancer in SLE has been recently suggested (SIR: 1.75, 95\% CI: 1.14-2.64) [17]. Numerous studies have reported associations between SLE and thyroid auto-immunity [18], which itself is associated with an increased thyroid cancer risk [19]. It was noted in one case-control study that a higher risk of papillary thyroid cancer was seen in SLE, especially for patients with thyroid autoimmunity [20]. However, thyroid cancers remain relatively uncommon events, even in SLE. In the updated multicenter cohort study conducted by our group [5], only 24 cases occurred in 15,980 patients observed for 119,846 person-years (representing approximately one event in every 5000 person-years).

\section{Cervical cancer \& dysplasia}

Several studies have shown that women with SLE were found to have an increased frequency of abnormal cervical Papanicolaou (Pap) smears compared with the general population [21]. A cohort analysis of lupus patients in the Wayne State University Lupus Registry indicated that women with lupus were 3.5-times more likely to develop high-grade cervical dysplasia or cervical cancer than the controls [22]. Published data have not emphasized an increase in invasive cervical cancers in SLE, compared with the general population [23], but it is not a common malignancy and single-center studies often cannot represent the risk of malignancy with precision. Interestingly, emerging data suggest a higher risk of vulvar cancers in SLE [17]. In both cervical dysplasia and vulvar cancers, an important factor is the possibility of altered clearance of human papillomavirus (HPV). A notably high risk of HPV infection appears in at least some individuals with SLE [24,25].

Regarding other risks for cervical dysplasia, it is recognized that exposure to immunosuppressants in SLE may have a particular influ-ence, especially cyclophosphamide [26,27] and potentially other agents such as azathioprine. In this context, it is important to keep in mind that the American College of Obstetrics and Gynecology Cervical Cancer Screening Guidelines recommend a cervical cytology screening every 2 years in the general population and every year in immunosuppressed patients [28]. Screening is especially required in women who have had a previous abnormal pap result and/or risk factors for HPV infection [29-32].

However, in women with chronic diseases such as SLE, screening is often neglected; some of the physicians working in our research group note that in some countries, including the UK, the availability of gynecology resources may create difficulties in ensuring that women are screened more often than every 3 years [33]. According to one study, patients with SLE with the most severe disease (based on SLE/ACR damage index scores) were the least likely to have undergone cervical screening [34]. Thus, specialists who follow female SLE patients should try to ensure that these individuals have access to a practitioner who can coordinate this aspect of care, and at least try to follow national guidelines. 


\section{Do drugs cause cancer in SLE?}

Studies have found that many of the patients with SLE who are diagnosed with malignancies (and/or die from) have not been exposed to immunosuppressive drugs [35-38]. Other studies, largely based on increased cancer risk in nonlupus renal transplant patients (who are also treated with immunosuppressant agents) have, for a long time, created a concern that much of the excess cancer risk in SLE is due to immunosuppressive use [39]. However, increasing evidence suggests this may not be so.

A recent small case-control study from Sweden compared 16 cases of NHL arising in SLE with 26 cancer-free control patients with SLE (matched on observation time and sex). The study evaluated azathioprine or cyclophosamide exposure but did not produce precise estimates of the effects of exposure [38]. Estimates adjusted for disease activity were also not provided, thus the conclusions drawn from the study are limited. The crude relative risk of NHL after cyclophosphamide exposure was 1.1 (95\% CI: 0.3-3.3) and after azathioprine was 0.9 (95\% CI: $0.5-2.5$ ). In this SLE population, only a small minority (two out of 18) of myeloid leukemia cases had ever received azathioprine or cyclophosphamide [40].

A larger scale multicenter case-cohort study, combining leukemia and lymphoma in the analyses of hematological cancers, produced adjusted drug-specific hazard ratios (estimates) using a lag period of 5 years postexposure (since malignancies often only arise years after a drug exposure) [41]. Adjustment for demographics, disease duration and lupus damage produced a hazard ratio for cyclophosphamide of 2.55 (95\% CI: $0.94-13.37$ ); for azathioprine, 1.02 (95\% CI: 0.34-33.03); and for methotrexate, 2.57 (95\% CI: 0.80-88.27).

The lack of precise results has prompted further attempts to differentiate the effects of lupus activity versus treatments in an ongoing case-cohort study of lymphoma risk in SLE. To date, preliminary results, controlling for disease activity, support a possible increased risk of lymphoma in cyclophosphamide users, without definitively demonstrating an increased risk for any other medications (adjusted for demographics and disease activity, the odds ratio for cyclophosphamide was $1.99,95 \%$ CI: 1.00-3.96) [42]. More detailed analyses specifically looking at dose, routes and scheduling are underway.

Antimalarials have been proposed to have potential use in cancer treatment [43], possibly through a cell death process called autophagy [44]. In the case-cohort analyses indicated above [38,41], antimalarials did not show definite protective effects against cancer risk. However, it remains possible that certain drug exposures in SLE may be beneficial with respect to cancer risk, as they may actually protect against carcinogenesis. For example, aspirin and NSAIDs are known to be potentially protective for a number of cancers [44,45]; corticosteroids may also alter cancer risk [46]. However, further study involving large-scale collaboration would be required to confirm the hypotheses that drug exposure could reduce the incidence of certain cancers.

\section{Decreased cancer risk in SLE}

Data from a previous study of cancer in SLE have shown a considerable decreased risk of hormone-sensitive cancers [4,5]. These cancers include breast cancer (SIR: 0.70, 95\% CI: 0.58-0.85), endometrial cancer (SIR: 0.49, 95\% CI: 0.27-0.83) and ovarian cancer (SIR: $0.56,95 \%$ CI: 0.28-0.97) in women. Data pooled from four studies of large SLE cohorts suggest that prostate cancer risk in males with SLE is also decreased (SIR: 0.72, 95\% CI: $0.57-0.89$ ) [7]. This lower risk may be due to hypoadrenergic states, which may occur in men with SLE, that modify the androgen pathways. 
The reduced risk in females with SLE, related to breast and other hormone-sensitive cancer, could potentially be attributed to alterations in the metabolism of estrogen and/or other hormones in women with SLE. In that event, one might anticipate that cancer risk would only be decreased in premenopausal women with SLE, not in postmenopausal SLE. However, in sensitivity analyses of the SLE cohort data [5], stratifying female subjects according to age ( $<50$ years, mainly premenopausal, and $\geq 50$ years, mainly postmenopausal) showed decreased breast cancer risk in SLE (relative to the general population) for both age groups.

One area of potential study is that the decreased incidence of certain cancers in SLE could be correlated to specific genetic factors that put an individual at risk for SLE, but protect against things such as breast cancer. To test this hypothesis, we explored whether the single nucleotide polymorphisms (SNPs) predisposing to SLE might be protective against breast cancer (in women in the general population), using data from a large breast cancer genomewide association study [47]. Within this large breast cancer data set, we did not demonstrate important associations with ten lupus-associated SNPs, and an inverse risk of breast cancer. Thus, if decreased breast cancer risk in SLE is influenced by genetic profiles, this may be due to complex interactions and/or epigenetic factors, or SNPs not examined in our study.

\section{Conclusion}

Cancer risk in SLE is an important issue to consider. The risk of NHL and certain other cancers is increased in SLE versus the general population. Regarding some of the immunosuppressive drugs used to treat SLE, there is little evidence suggesting that drugs are the main risk factor for lymphoma, although cyclophosphamide might serve as a trigger in some cases. Further work must be carried out to determine the exact mechanisms (potentially hormonal) behind the altered risk of cancer in SLE. Examining SLE patients can lead to the risk of unwanted variability, as SLE is generally assessed based on the experience of the treating physician [48]. In order to reduce this potential for variability, the European League Against Rheumatism (EULAR) has developed recommendations for monitoring SLE patients to help create a more uniform assessment [49]. In terms of cancer screening, these recommendations suggest that patients with SLE should at least follow cancer screening recommended for the general population. Furthermore, due to the epidemiological and clinical characteristics of patients with SLE, the implementation of SLE-specific guidelines is under consideration for the near future [49].

An emerging finding is the decreased risk of certain nonhematologic cancers (such as breast cancer) for lupus patients. Although encouraging, this should not prevent women with SLE from continuing with mammogram screening programs as recommended for the general population, since breast cancer will still be the most common cancer that women with SLE will ever get.

\section{Future perspective}

Although much has been learnt over the past decade regarding cancer risk in SLE, much yet remains to be discovered. Recent advances acknowledged in this review provide an incentive for further research into this field and emphasize the need for development of more effective and safer drugs for lupus. Furthermore, it is important that patients with SLE undergo preventative measures, including smoking cessation and regular cancer screening programs (as per established guidelines for the general population), especially for cervical cancers (recommended yearly in immunosuppressed patients). 


\section{References}

Papers of special note have been highlighted as:

- of interest

- of considerable interest

1. Bernatsky S, Boivin J, Lawrence J, et al. An international cohort study of cancer in systemic lupus erythematosus. Arthritis Rheum. 2005; 52:1481-1490. [PubMed: 15880596]

2. Parikh-Patel A, White RH, Allen M, Cress R. Cancer risk in a cohort of patients with systemic lupus erythematosus (SLE) in California. Cancer Causes Control. 2008; 19:887-894. [PubMed: 18386139]

3. Mellemkjaer L, Andersen V, Linet MS, Gridley G, Hoover R, Olsen JH. Non-Hodgkin's lymphoma and other cancers among a cohort of patients with systemic lupus erythematosus. Arthritis Rheum. 1997; 40:761-768. [PubMed: 9125261]

4. Bjornadal L, Lofstrom B, Yin L, Lundberg IE, Ekbom A. Increased cancer incidence in a Swedish cohort of patients with systemic lupus erythematosus. Scand J Rheumatol. 2002; 31:66-71.

[PubMed: 12109649]

5-.. Bernatsky S, Clarke A, Petri MA, et al. Further defining cancer risk in systemic lupus: updated results in an expanded international multi-centre cohort. Arthritis Rheum. 2010; 62:S731. Updated cohort study based on recent data regarding cancer in patients with systemic lupus erythematosus (SLE).

6r. Bernatsky S, Ramsey-Goldman R, Foulkes W, Gordon C, Clarke A. Breast, ovarian, and endometrial malignancies in systemic lupus erythematosus: a meta-analysis. Br J Cancer. 2010; 104:1478-1481. Novel meta-analysis focusing on the issue of cancer occurrence in women with SLE. [PubMed: 21487409]

7-. Bernatsky S, Ramsey-Goldman R, Gordon C, Clarke AE. Prostate cancer in systemic lupus erythematosus. Int J Cancer. 2011; 129(12):2966-2969. Highlighting the decreased incidence of prostate cancer in men with SLE. [PubMed: 21448902]

8. Bernatsky S, Boivin J, Manzi S, et al. Mortality in systemic lupus erythematosus. Arthritis Rheum. 2006; 54:2550-2557. [PubMed: 16868977]

9. Bernatsky S, Ramsey-Goldman R, Rajan R, et al. Non-Hodgkin's lymphoma in systemic lupus erythematosus. Ann Rheum Dis. 2005; 64:1507-1509. [PubMed: 16162903]

10. Ali YM, Urowitz MB, Ibanez D, Gladman DD. Monoclonal gammopathy in systemic lupus erythematosus. Lupus. 2007; 16:426-429. [PubMed: 17664233]

11. Ott G, Rosenwald A. Molecular pathogenesis of follicular lymphoma. Haematologica. 2008; 93:1773-1776. [PubMed: 19050067]

12. Habbeth OA, Lorvik KB, Hammarström C, et al. Inflammation driven by tumour-specific Th1 cells protects against B-cell cancer. Nat Commun. 2011; 2:240. [PubMed: 21407206]

13. Rossi E, Catania G, Truini M, Ravetti G, Grassia L, Marmont A. Patients with systemic lupus erythematosus (SLE) having developed malignant lymphomas. Complete remission of lymphoma following high-dose chemotherapy, but not of SLE. Clin Exp Rheumatol. 2011; 29:555-559. [PubMed: 21722503]

14. Hemminki K, Liu X, Ji J, Sundquist J, Sundquist K. Effect of autoimmune diseases on risk and survival in histology-specific lung cancer. Eur Respir J. 2012; 40(6):1489-1495. [PubMed: 22323571]

15. Bin J, Bernatsky S, Gordon C, et al. Lung cancer in systemic lupus erythematosus. LungCancer. 2007; 56:303-306.

16. Adzić TN, Pesut DP, Nagorni-Obradović LM, Stojsić JM, Vasiljević MD, Bouros D. Clinical features of lung cancer in patients with connective tissue diseases: a 10-year hospital based study. Respir Med. 2008; 102(4):620-624. [PubMed: 18178071]

17-r. Bernatsky S, Ramsey-Goldman R, Labrecque J, et al. Cancer risk in systemic lupus: an updated international multi-centre cohort study. J Autoimmunity. 2013 10.1016/j. jaut.2012.12.009. (Epub ahead of print). Most recent data related to cancer risk in SLE. 
18. Antonelli A, Fallahi P, Mosca M, et al. Prevalence of thyroid dysfunctions in systemic lupus erythematosus. Metabolism. 2010; 59(6):896-900. [PubMed: 20005534]

19. Azizi G, Malchoff CD. Autoimmune thyroid disease: a risk factor for thyroid cancer. Endocr Pract. 2011; 17(2):201-209. [PubMed: 20841310]

20-. Antonelli A, Mosca M, Fallahi P, et al. Thyroid cancer in systemic lupus erythematosus: a casecontrol study. J Clin Endocrinol Metab. 2010; 95(1):314-318. Important recent study of thyroid cancer in SLE. [PubMed: 19906791]

21. Papadimitraki, E.; Bertsias, G.; Chamilos, G.; Boumpas, D. Systemic lupus erythematosus: cytotoxic agents. In: Tsokos, G.; Buyon, J.; Koike, T.; Lahita, R., editors. Systemic Lupus Erythematosus. 5. Academic Press; San Diego, CA, USA: 2011. p. 1083-1108.

22. Dhar JP, Essenmacher L, Ager J, Sokol RJ. Ominous cervical cytopathology in women with lupus. Int J Gynecol Obstet. 2005; 89(3):295-296.

23. Santana IU, Gomes AD, Lyrio LD, Rios Grassi MF, Santiago MB. Systemic lupus erythematosus, human papillomavirus infection, cervical pre-malignant and malignant lesions: a systematic review. Clin Rheumatol. 2011; 30:665-672. Recent look at the issue of human papillomavirus, cervical dysplasia and SLE. [PubMed: 21072553]

24. Nath R, Mant C, Luxton J, et al. High risk of human papillomavirus type 16 infections and of development of cervical squamous intraepithelial lesions in systemic lupus erythematosus patients. Arthritis Rheum. 2007; 57:619-625. [PubMed: 17471531]

25. Lee YH, Choe JY, Park SH, et al. Prevalence of human papilloma virus infections and cervical cytological abnormalities among Korean women with systemic lupus erythematosus. J Korean Med Sci. 2010; 25:1431-1437. [PubMed: 20890422]

26. Klumb EM, Araujo ML Jr, Jesus GR, et al. Is higher prevalence of cervical intraepithelial neoplasia in women with lupus due to immunosuppression? J Clin Rheumatol. 2010; 16:153-157. [PubMed: 20407390]

27. Bernatsky S, Ramsey-Goldman R, Gordon C, et al. Factors associated with abnormal Pap results in systemic lupus erythematosus. Rheumatology (Oxford). 2004; 43:1386-1389. [PubMed: $15280571]$

28. Liu H, Ding Q, Yang K, Zhang T, Li G, Wu G. Meta-analysis of systemic lupus erythematosus and the risk of cervical neoplasia. Rheumatology. 2011; 50(2):343-348. [PubMed: 20929972]

29. Blumenfeld Z, Lorber M, Yoffe N, Scharf Y. Systemic lupus erythematosus: predisposition for uterine cervical dysplasia. Lupus. 1994; 3:59-61. [PubMed: 8025589]

30. Bateman H, Yazici Y, Leff L, Peterson M, Paget SA. Increased cervical dysplasia in intravenous cyclophosphamide-treated patients with SLE: a preliminary study. Lupus. 2000; 9:542-544. [PubMed: 11035421]

31. Dhar JP, Kmak D, Bhan R, Pishorodi L, Ager J, Sokol RJ. Abnormal cervicovaginal cytology in women with lupus: a retrospective cohort study. Gynecol Oncol. 2001; 82:4-6. [PubMed: 11426953]

32. Nyberg G, Eriksson O, Westberg NG. Increased incidence of cervical atypia in women with systemic lupus erythematosus treated with chemotherapy. Arthritis Rheum. 1981; 24:648-650. [PubMed: 7236321]

33. Gakidou E, Nordhagen S, Obermeyer Z. Coverage of cervical cancer screening in 57 countries: low average levels and large inequalities. PLoS Med. 2008; 5(6):e132. [PubMed: 18563963]

34. Bernatsky SR, Cooper GS, Mill C, Ramsey-Goldman R, Clarke AE, Pineau CA. Cancer screening in patients with systemic lupus erythematosus. J Rheumatol. 2006; 33:45-49. [PubMed: 16331804]

35. Moss KE, Ioannou Y, Sultan SM, Haq I, Isenberg DA. Outcome of a cohort of 300 patients with systemic lupus erythematosus attending a dedicated clinic for over two decades. Ann Rheum Dis. 2002; 61:409-413. [PubMed: 11959764]

36. Cibere J, Sibley J, Haga M. Systemic lupus erythematosus and the risk of malignancy. Lupus. 2001; 10:394-400. [PubMed: 11434573]

37. Pettersson T, Pukkala E, Teppo L, Friman C. Increased risk of cancer in patients with systemic lupus erythematosus. Ann Rheum Dis. 1992; 51:437-439. [PubMed: 1586239] 
38. Lofstrom B, Backlin C, Sundstrom C, Ekbom A, Lundberg IE. A closer look at non-Hodgkin's lymphoma cases in a national Swedish systemic lupus erythematosus cohort: a nested case-control study. Ann Rheum Dis. 2007; 66:1627-1632. [PubMed: 17517757]

39. Caillard S, Dharnidharka V, Agodoa L, Bohen E, Abbott K. Posttransplant lymphoproliferative disorders after renal transplantation in the United States in era of modern immunosuppression. Transplantation. 2005; 80(9):1233-1243. [PubMed: 16314791]

40. Lofstrom B, Backlin C, Sundstrom C, Hellstrom-Lindberg E, Ekbom A, Lundberg IE. Myeloid leukaemia in systemic lupus erythematosus - a nested case-control study based on Swedish registers. Rheumatology (Oxford). 2009; 48:1222-1226. [PubMed: 19608725]

41. Bernatsky S, Boivin JF, Joseph L, et al. The relationship between cancer and medication exposures in systemic lupus erythematosus: a case-cohort study. Ann Rheum Dis. 2008; 67:74-79. [PubMed: 17545189]

42. Bernatsky S, Clarke A, Labreque J, et al. Lymphoma risk in systemic lupus: effects of disease activity versus treatment. Arthritis Rheum. 2012; 63(10S):S25791.

43. Ruiz-Irastorza G, Ugarte A, Egurbide MV, et al. Antimalarials may influence the risk of malignancy in systemic lupus erythematosus. Ann Rheum Dis. 2007; 66:815-817. [PubMed: 17204564]

44. Amaravadi RK. Autophagy-induced tumor dormancy in ovarian cancer. J Clin Invest. 2008; 118:3837-3840. [PubMed: 19033653]

45. Rahim R, Strobl JS. Hydroxychloroquine, chloroquine, and all-trans retinoic acid regulate growth, survival, and histone acetylation in breast cancer cells. Anticancer Drugs. 2009; 20:736-745. [PubMed: 19584707]

46. Moran EM. Epidemiological and clinical aspects of nonsteroidal anti-inflammatory drugs and cancer risks. J Environ Pathol Toxicol Oncol. 2002; 21:193-201. [PubMed: 12086406]

47. Bernatsky S, Easton D, Dunning A, et al. Decreased breast cancer risk in systemic lupus erythematosus: the search for a genetic basis continues. Lupus. 2012; 21(8):896-899. [PubMed: 22495874]

48-. Mosca M, Tani C, Aringer M, et al. Development of quality indicators to evaluate the monitoring of SLE patients in routine clinical practice. Autoimmun Rev. 2011; 10:383-388. Recent paper on quality indicators in SLE. [PubMed: 21224016]

49. Mosca M, Tani C, Aringer M, et al. European League Against Rheumatism recommendations for monitoring patients with systemic lupus erythematosus in clinical practice and in observational studies. Ann Rheum Dis. 2010; 69:1269-1274. [PubMed: 19892750] 


\section{Executive summary}

\section{Background}

- Cancer risk profiles in systemic lupus erythematosus (SLE) have become much better understood in the past 10 years; however, much remains to be discovered.

\section{Do drugs cause cancer in SLE?}

- Immunosuppressive drugs have been suggested to play a role in the increased risk of cancer in SLE, but there may also be other important factors, including SLE activity itself.

\section{Decreased cancer risk in SLE}

- It remains possible that certain drug exposures in SLE may be beneficial with respect to cancer risk, as they may actually protect against carcinogenesis with respect to certain cancer types, such as breast cancer.

\section{Preventive measures}

- Preventive measures, such as smoking cessation and regular cancer screening, are essential for patients with SLE. 